\title{
From Classical to Quantum Nonlinear Photonic Mixers on Silicon Microchips (Project Report 1201308-Y4)
}

\section{SHAYAN MOOKHERJEA}

Department of Electrical and Computer Engineering, University of California, San Diego, MC 0407 La Jolla CA 92093-0407 USA

Email:smookher@ucsd.edu

\section{Introduction and Goals}

The main goal of this NSF-funded project [1201308 - Year 4] is to develop an integrated photonics device technology based on silicon photonics which can be used for compact and efficient nonlinear classical and quantum photonics applications.

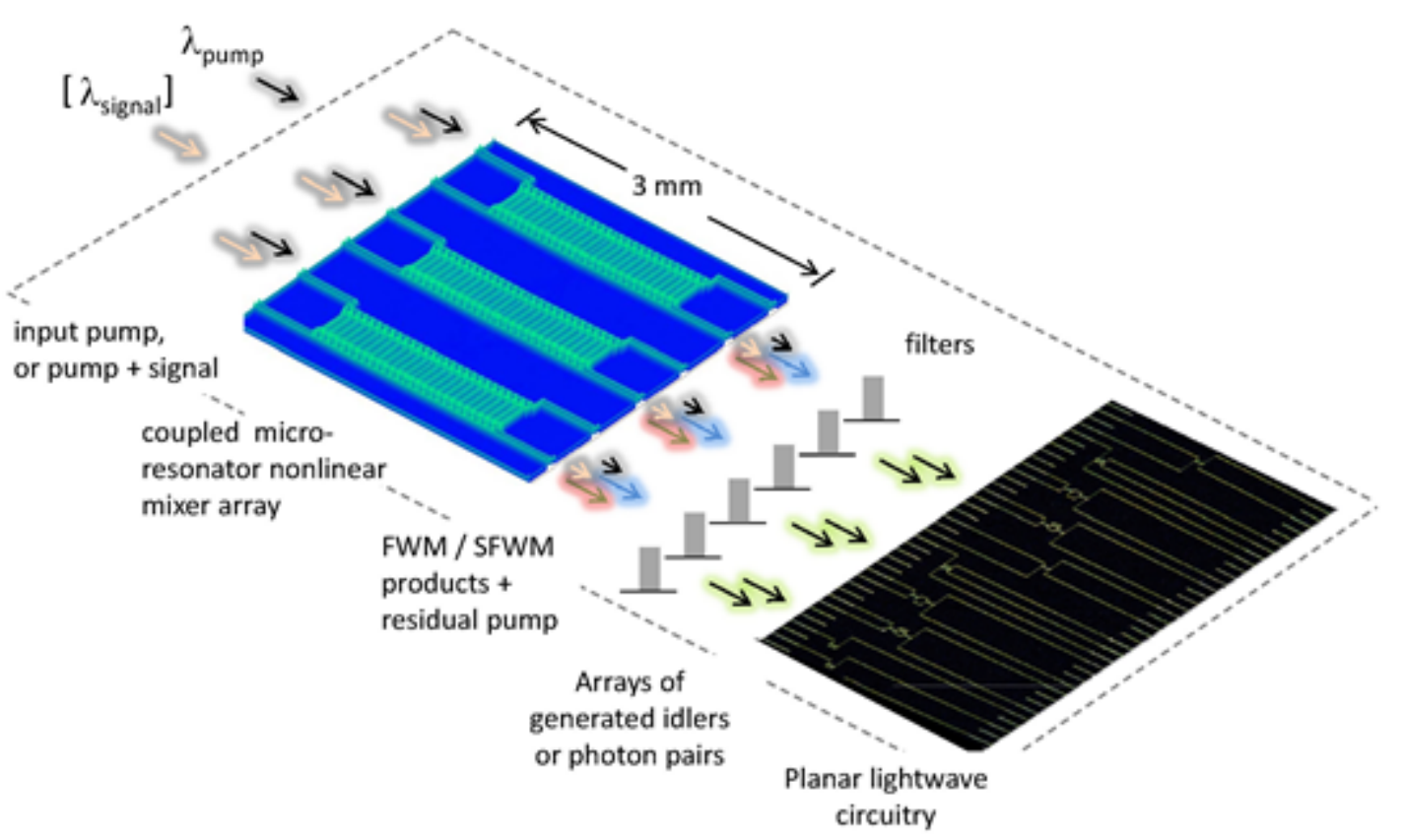

Figure 1 Vision of a monolithic silicon chip (dotted outline) utilizing the proposed mixer (shown in blue), which can be used for telecom-band four-wave mixing for data translation or ultrafast switching, infrared generation, biphoton and heralded single photon source, chip-scale quantum photonics with the incorporation of planar lightwave circuitry as indicated, and other applications, to replace entire table-top or rack-sized assemblies

Fig. 1 shows a conceptual drawing of an integrated photonic circuit which contains several mixer devices and used in a quantum photonics context. Similar to the widely-used radio-frequency mixers, optical mixers can generate new frequencies (wavelengths) by transferring power from other input waves. In silicon photonics, the foundational process for this type of interaction is fourwave mixing, in which one or more input pump and signal waves interact using 
the chi-(3) nonlinearity to generate idler waves. Alternatively, if the input is only the pump, then energy-conversation and phase-matching require that correlated photons are produced at the signal and idler wavelengths. This project studies wavelength conversion in waveguides, microring resonators and coupled-resonator optical waveguides (CROWs) [1], which may also be useful for filters [2] and lasers [3], [4], among other types of photonic devices such as entangled photon-pair generation devices and filters (see Year 3 report).

\section{Activities}

We demonstrated photon-pair generation from a high-Q Si microring resonator over a wide temperature range. One of the practical issues with using silicon photonic micro-resonators as photon-pair sources is that the resonance wavelength shifts easily with temperature. Although the range of resonance shift can be compensated by the tuning range of a typical DFB laser diode, the resonance has to be tracked continuously. Unless carefully designed, incorporating additional splitters or filters in the optical pathway incur loss, which reduces the brightness and utility of the pair source. We can observe the optically-pumped microring resonator with an infrared camera to verify that the pump remains resonant during the pair-generation process [5], [6]. We also presented an all-silicon approach to monitoring the temperature-induced wavelength shifts during photon-pair generation, and thereby achieved stable pair generation over a temperature variation of more than $30^{\circ} \mathrm{C}$ [7].

We addressed the issue of generating short pulses for improving the spectral properties of photon pairs generated by silicon microring resonators. In order to achieve a closer-to-circular joint spectral intensity (JSI), some researchers have used mode-locked lasers to achieve the necessary (few tens of picoseconds) duration optical pump pulses [8]. Mode-locked lasers (MLL's) are big, expensive and costly lab instruments. In a physics experiment, using crystals or fibers for pair generation would add little to the cost or size of the overall instrument when using MLL's anyway. In contrast, Si microchips are popular because they are inexpensive and compact. We demonstrated pulsed pumping of the SFWM process by using just a CW laser diode, and an electronic component to carve pulses into the pump light by using an off-theshelf electro-optic modulator (EOM) [9]. The $\mathrm{CW}$ laser diode, the electronic driver component and the EOM are all inexpensive, compact components. This helps simplify the technology of pair generation using Si micro-photonic chips and helps make the source small and inexpensive.

Challenges: Photon-pair generation depends critically on phase matching since there is only one optical input (the pump). Phase matching, in turn, depends on the dispersion properties of the waveguides and the devices formed using waveguides. In silicon photonics, waveguides and optical components such as 
directional couplers have a higher dispersion than in silica planar lightwave [10]. An accurate design of the waveguide-resonator coupling is important and often requires time-consuming simulations, since there can be significant deviations from conventional coupled-mode theory in the high-index-contrast regime [11]. The coupling coefficient determines many properties of the CROW structure, including bandwidth, and the robustness to disorder.

Another area of potential concern is nonlinearity within the directional coupler itself. The optical pump power can be increased in an attempt to improve the brightness of photon-pair generation. However, this can sometimes affect the transmission function of resonators, especially racetrack resonators, in way that leads to bistability and oscillatory behavior [12]. For the parameter ranges of interest in this project, we were not adversely impacted by the bistable regime of operation in the measurements that we performed.

The silicon microring CROW devices which we used in our experiments were designed using racetrack resonators that consist of both straight and curved waveguide sections. Compared to conventional waveguides, a CROW provides significant resonant enhancement of the FWM conversion efficiency [13], [14], and also offers a relatively wide passband (up to several nanometers). The abinitio simulation of such devices is highly time-consuming, but a useful preliminary design can be made using analytical derivations. A study of pulse propagation shows the helpful role which slow light plays in enhancing mixing properties [15], [16]. In our previous research, low loss CROWs were successfully fabricated and measured, and a good agreement was achieved between experiments and theory [17]-[19].

It is inevitable that foundry-fabricated silicon photonics will have some degree of fabrication disorder. We considered a number of ideas such as postfabrication trimming using high-resolution experimental techniques which we had previously developed [5],[6]. However, for this project, it was adequate to use thermal tuning of the devices to the target wavelength.

Because of the unconventional nature of optical propagation [22]-[24], disorder in CROWs can manifest a range of complex physical behavior including localization of light. The physics of localization are usually studied in dielectric powders and other static devices, which cannot be controlled at microsecond or faster time scales. In contrast, devices fabricated in silicon photonics can sometimes be controlled electronically at gigahertz rates [25]. A deeper understanding of the transition between localization and extended propagation regimes is interesting from both a fundamental and applied perspective. 
More separable (heralding)

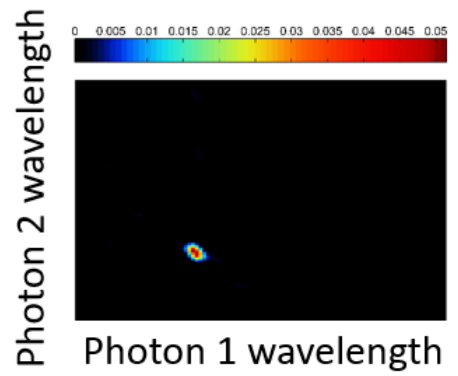

same chip

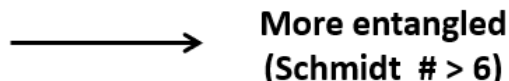

(Schmidt \# > 6)

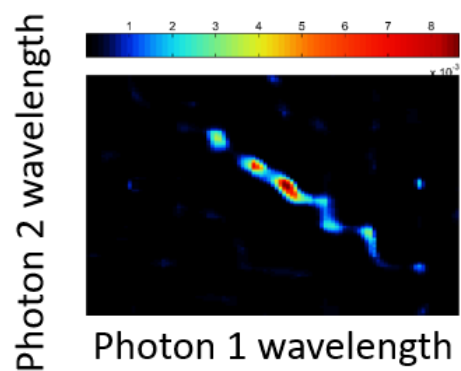

Figure 2 The same CROW device can generate different bi-photon joint-spectral intensities depending on the placement of the pump within the passband.

\section{Progress and Impact}

Most pair-generation devices are pre-configured for one type of nonlinear interaction. However, CROWs can be dynamically programmed to generate a variety of biphoton wavefunctions, as shown by the measured joint spectral intensity (JSI) shown in Fig. 2 The controllable entanglement properties of the photon pairs generated by the CROW device were experimentally verified [26], [27]. There are interesting differences in the SFWM process in single rings and coupled rings, as shown in Fig. 3. Because of the coupling between the resonators, the transmission shows a richer set of physical behavior with $\mathrm{N}$ different supermodes in a chain of $\mathrm{N}$ resonators. The overlap of the transmission miniband peaks with the phase-matching and pump spectral properties determines the two-photon state and the associated JSI and Schmidt number $(\mathrm{K})$, which can range from a quasi-separable state with $\mathrm{K}$ approximately equal to 1 , to a highly-entangled state with $\mathrm{K}$ much greater than 1 .

As shown in Fig. 1, the eventual goal is to incorporate mixers and filters on the same chip. In the domain of classical optics, we showed the combination of an on-chip ring mixer and a tunable filter last year [28]. Quantum light generation on an integrated photonic chip is now a topic of considerable interest [29]. However, in this regime, the filtering requirements are much more stringent and complete integration of the pair source could not be achieved within the duration of this project. 


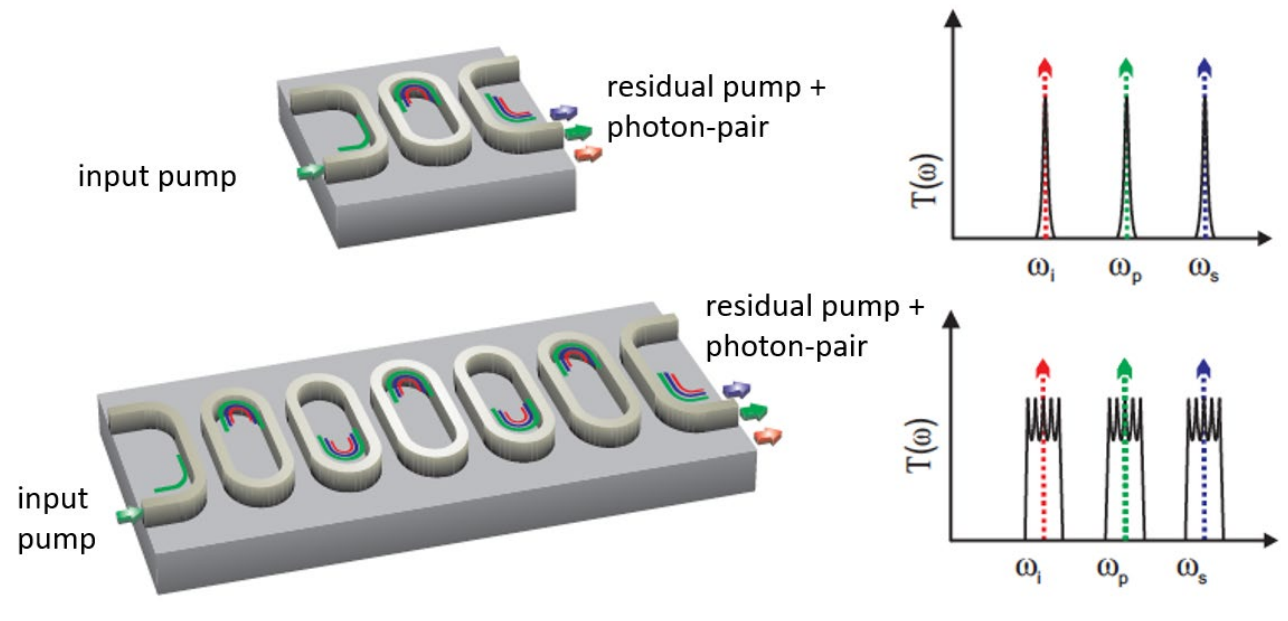

Figure 3 The difference between SFWM in a single microring resonator and in a coupledresonator optical waveguide (CROW).

We have already studied classical FWM experimentally in waveguides, microrings and coupled-resonator waveguides [30]. We showed improved FWM conversion efficiency and absolute conversion in both silicon waveguides and in resonators in which a p-i-n diode was fabricated across the waveguide cross-section to sweep out electronic free carriers that were generated a higher pump power levels [31].

Rapidly-tunable wavelength conversion is a topic of growing interest. Compared to slow thermo-optic tuning [32], all-optical frequency conversion can achieve a much faster response, but requires fast control over the optical pump beams, which is not easy. For relatively short frequency ranges (about $\pm 100 \mathrm{GHz}$, which spans a couple of DWDM channels), frequency conversion can be achieved more simply by using integrated electro-optic modulators, which were demonstrated in subsequent research [33].

Impact on Human Resources: NSF funds supported two graduate students (both of whom defended their $\mathrm{PhD}$ thesis on topics related to this project), one postdoctoral scholar and two undergraduate students. They worked on device design, fabrication and testing. In addition to publishing our results in peerreviewed journals and conferences, we presented our work at invited seminars at several national and international venues.

\section{Subsequent Extensions}

The second-order chi-(2) nonlinearity of some materials such as lithium niobate [34] is stronger than the third-order chi-(3) nonlinearity of silicon, but requires 
a non-traditional fabrication approach in order to incorporate it as part of more complex lightwave circuits. Meanwhile, it is also possible to achieve an improved level of performance from silicon photonics itself, by careful and systematic optimization of the pair-generation properties of silicon microring resonators [35]. We anticipate that the development of a practical entanglement pair source made using scalable silicon manufacturable technology may be a significant advancement for areas such as detector calibration, precision measurement, secure communications and quantum networks. However, the transition of novel photonics hardware into a manufactured chip-scale technology intended for practical applications will take time and resources in prototyping, packaging and testing.

\section{Open-Access Reporting Initiative}

PRAISE: This open-access document is provided in support of our PRAISE

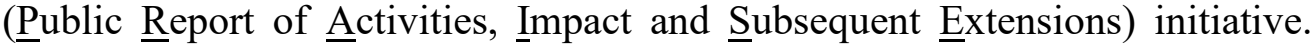
What is it? An open-access document shared with the public which describes the research outcomes of publicly-funded projects. For us, these projects are typically funded by the NSF (National Science Foundation).

\section{References}

[1] F. Morichetti, C. Ferrari, A. Canciamilla, and A. Melloni, "The first decade of coupled resonator optical waveguides: bringing slow light to applications," Laser \& Photon. Rev., vol. 6, no. 1, pp. 74-96, Jan. 2012, doi: 10.1002/lpor.201100018.

[2] S. Mookherjea, "Spectral characteristics of coupled resonators," J. Opt. Soc. Am. B, vol. 23, no. 6, p. 1137, Jun. 2006, doi: 10.1364/JOSAB.23.001137.

[3] S. Mookherjea, "Semiconductor coupled-resonator optical waveguide laser," Appl. Phys. Lett., vol. 84, no. 17, pp. 3265-3267, Apr. 2004, doi: 10.1063/1.1719278.

[4] S. Mookherjea, "Using gain to tune the dispersion relation of coupledresonator optical waveguides," IEEE Photon. Technol. Lett., vol. 18, no. 5, pp. 715-717, Mar. 2006, doi: 10.1109/LPT.2006.871144.

[5] M. L. Cooper, G. Gupta, J. S. Park, M. A. Schneider, I. B. Divliansky, and S. Mookherjea, "Quantitative infrared imaging of silicon-on-insulator microring resonators," Opt. Lett., vol. 35, no. 5, p. 784, Mar. 2010, doi: 10.1364/OL.35.000784.

[6] S. Mookherjea and H. R. Grant, "High dynamic range microscope infrared imaging of silicon nanophotonic devices," Opt. Lett., vol. 37, no. 22, p. 4705, Nov. 2012, doi: 10.1364/OL.37.004705. 
[7] R. Kumar, J. R. Ong, J. Recchio, K. Srinivasan, and S. Mookherjea, "Spectrally multiplexed and tunable-wavelength photon pairs at $155 \mu \mathrm{m}$ from a silicon coupled-resonator optical waveguide," Opt. Lett., vol. 38, no. 16, p. 2969, Aug. 2013, doi: 10.1364/OL.38.002969.

[8] O. Alibart, D. B. Ostrowsky, P. Baldi, and S. Tanzilli, "High-performance guided-wave asynchronous heralded single-photon source," Opt. Lett., vol. 30, no. 12, pp. 1539-1541, 2005.

[9] M. Savanier and S. Mookherjea, "Generating photon pairs from a silicon microring resonator using an electronic step recovery diode for pump pulse generation," Appl. Phys. Lett., vol. 108, no. 25, p. 251102, Jun. 2016, doi: 10.1063/1.4954261.

[10] R. Aguinaldo, Yiran Shen, and S. Mookherjea, "Large Dispersion of Silicon Directional Couplers Obtained via Wideband Microring Parametric Characterization," IEEE Photon. Technol. Lett., vol. 24, no. 14, pp. 1242-1244, Jul. 2012, doi: 10.1109/LPT.2012.2198639.

[11] M. L. Cooper and S. Mookherjea, "Numerically-assisted coupledmode theory for silicon waveguide couplers and arrayed waveguides," Opt. Express, vol. 17, no. 3, p. 1583, Feb. 2009, doi: 10.1364/OE.17.001583.

[12] S. Mookherjea and M. A. Schneider, "The nonlinear microring adddrop filter," Opt. Express, vol. 16, no. 19, p. 15130, Sep. 2008, doi: 10.1364/OE.16.015130.

[13] S. Mookherjea and A. Yariv, "Second-harmonic generation with pulses in a coupled-resonator optical waveguide," Phys. Rev. E, vol. 65, no. 2, p. 026607, Jan. 2002, doi: 10.1103/PhysRevE.65.026607.

[14] S. Mookherjea and A. Yariv, "Kerr-stabilized super-resonant modes in coupled-resonator optical waveguides," Phys. Rev. E, vol. 66, no. 4, p. 046610, Oct. 2002, doi: 10.1103/PhysRevE.66.046610.

[15] S. Mookherjea and A. Yariv, "Optical pulse propagation in the tightbinding approximation," Opt. Express, vol. 9, no. 2, p. 91, Jul. 2001, doi: 10.1364/OE.9.000091.

[16] S. Mookherjea and A. Yariv, "Optical pulse propagation and holographic storage in a coupled-resonator optical waveguide," Phys. Rev. $E$, vol. 64, no. 6, p. 066602 , Nov. 2001, doi: 10.1103/PhysRevE.64.066602.

[17] M. L. Cooper et al., "235-ring Coupled-Resonator Optical Waveguides," in Conference on Lasers and Electro-Optics 2010, San Jose, California, 2010, p. CTuHH3. doi: 10.1364/CLEO.2010.CTuHH3.

[18] S. Mookherjea and M. A. Schneider, "Avoiding bandwidth collapse in long chains of coupled optical microresonators," Opt. Lett., vol. 36, no. 23, p. 4557, Dec. 2011, doi: 10.1364/OL.36.004557.

[19] M. L. Cooper and S. Mookherjea, "Modeling of Multiband Transmission in Long Silicon Coupled-Resonator Optical Waveguides," 
IEEE Photon. Technol. Lett., vol. 23, no. 13, pp. 872-874, Jul. 2011, doi: 10.1109/LPT.2011.2141657.

[20] Y. Shen, I. B. Divliansky, D. N. Basov, and S. Mookherjea, "Electricfield-driven nano-oxidation trimming of silicon microrings and interferometers," Opt. Lett., vol. 36, no. 14, p. 2668, Jul. 2011, doi: 10.1364/OL.36.002668.

[21] Y. Shen, I. B. Divliansky, D. N. Basov, and S. Mookherjea, "Perfect set-and-forget alignment of silicon photonic resonators and interferometers," in Optical Fiber Communication Conference/National Fiber Optic Engineers Conference 2011, Los Angeles, California, 2011, p. PDPC3. doi: 10.1364/OFC.2011.PDPC3.

[22] S. Mookherjea and A. Yariv, "Pulse propagation in a coupled resonator optical waveguide to all orders of dispersion," Phys. Rev. E, vol. 65, no. 5, p. 056601, Apr. 2002, doi: 10.1103/PhysRevE.65.056601.

[23] M. L. Cooper et al., "Waveguide dispersion effects in silicon-oninsulator coupled-resonator optical waveguides," Opt. Lett., vol. 35, no. 18, p. 3030, Sep. 2010, doi: 10.1364/OL.35.003030.

[24] S. Mookherjea, D. S. Cohen, and A. Yariv, "Nonlinear dispersion in a coupled-resonator optical waveguide," Opt. Lett., vol. 27, no. 11, p. 933, Jun. 2002, doi: 10.1364/OL.27.000933.

[25] S. Mookherjea, J. R. Ong, X. Luo, and L. Guo-Qiang, "Electronic control of optical Anderson localization modes," Nature Nanotech, vol. 9, no. 5, pp. 365-371, May 2014, doi: 10.1038/nnano.2014.53.

[26] R. Kumar, M. Savanier, J. R. Ong, and S. Mookherjea, "Entanglement measurement of a coupled silicon microring photon pair source," Opt.

Express, vol. 23, no. 15, p. 19318, Jul. 2015, doi: 10.1364/OE.23.019318.

[27] R. Kumar, J. R. Ong, M. Savanier, and S. Mookherjea, "Controlling the spectrum of photons generated on a silicon nanophotonic chip," Nat Commun, vol. 5, no. 1, p. 5489, Dec. 2014, doi: 10.1038/ncomms6489.

[28] J. R. Ong, R. Kumar, and S. Mookherjea, "Silicon microring-based wavelength converter with integrated pump and signal suppression," Opt. Lett., vol. 39, no. 15, p. 4439, Aug. 2014, doi: 10.1364/OL.39.004439.

[29] J. R. Ong and S. Mookherjea, "Quantum light generation on a silicon chip using waveguides and resonators," Opt. Express, vol. 21, no. 4, p. 5171, Feb. 2013, doi: 10.1364/OE.21.005171.

[30] J. R. Ong et al., "Low-power continuous-wave four-wave mixing in silicon coupled-resonator optical waveguides," Opt. Lett., vol. 36, no. 15, pp. 2964-2966, 2011.

[31] J. R. Ong, R. Kumar, R. Aguinaldo, and S. Mookherjea, "Efficient CW Four-Wave Mixing in Silicon-on-Insulator Micro-Rings With Active Carrier Removal," IEEE Photon. Technol. Lett., vol. 25, no. 17, pp. 1699 1702, Sep. 2013, doi: 10.1109/LPT.2013.2272521. 
[32] R. Aguinaldo et al., "Wideband silicon-photonic thermo-optic switch in a wavelength-division multiplexed ring network," Opt. Express, vol. 22, no. 7, p. 8205, Apr. 2014, doi: 10.1364/OE.22.008205.

[33] X. Wang, P. O. Weigel, J. Zhao, M. Ruesing, and S. Mookherjea, "Achieving beyond-100-GHz large-signal modulation bandwidth in hybrid silicon photonics Mach Zehnder modulators using thin film lithium niobate," APL Photonics, vol. 4, no. 9, p. 096101, Sep. 2019, doi: 10.1063/1.5115243.

[34] J. Zhao, C. Ma, M. Rüsing, and S. Mookherjea, "High Quality Entangled Photon Pair Generation in Periodically Poled Thin-Film Lithium Niobate Waveguides," Phys. Rev. Lett., vol. 124, no. 16, p. 163603, Apr. 2020, doi: 10.1103/PhysRevLett.124.163603.

[35] C. Ma, X. Wang, V. Anant, A. D. Beyer, M. D. Shaw, and S. Mookherjea, "Silicon photonic entangled photon-pair and heralded single photon generation with CAR $>12,000$ and $\mathrm{g}^{\wedge}(2)(0)<0006$," Opt. Express, vol. 25, no. 26, p. 32995, Dec. 2017, doi: 10.1364/OE.25.032995. 